\title{
Treatment of Patients with High-Grade Aneurysmal Subarachnoid Hemorrhage
}

Can. J. Neurol. Sci. 2011; 38: 3

Management of patients with high-grade aneurysmal subarachnoid hemorrhage presents a therapeutic dilemma to treating physicians. Without aggressive treatment including early aneurysm repair and critical care measures to prevent or treat hydrocephalus, vasospasm, cerebral edema, and metabolic complications, those who survive the initial bleed will almost certainly succumb to recurrent hemorrhage or other complications. For those who present in poor neurological condition, the outcome may be extremely poor despite aggressive measures that use scarce resources and are expensive. On the other hand, a conservative treatment approach will almost certainly deny a small proportion of these patients the possibility of meaningful recovery.

In this issue of the CJNS, Diaz and Wong present the results of a selective approach to the management of patients presenting with high-grade subarachnoid hemorrhage. ${ }^{1}$ Their experience provides a glimmer of hope as well as some practical insights that can provide guidance to front-line clinicians. Over a tenyear-period, 33 patients with grade 4 and 5 subarachnoid hemorrhage were treated with early endovascular aneurysm repair and an aggressive medical protocol. It is important to note that this was a highly selected subgroup and does not represent a systematic review of all cases of high-grade SAH. By their estimate, the patients included in this retrospective series represents only $20-40 \%$ of the total number of high-grade $\mathrm{SAH}$ cases at their institution. Patients who had persistent coma or absent brainstem reflexes after initial resuscitation were not included, nor were those who had large clots causing mass effect or herniation, who were treated with urgent craniotomy to evacuate the hematoma and directly clip the ruptured aneurysm.

It is well known to those who manage SAH patients that some who present with coma will improve markedly with placement of a ventricular drain to treat hydrocephalus. Response to this simple intervention is probably one of the most important factors in the determination of management strategies for high-grade patients. In this series, as in others, ${ }^{2-4}$ improvement after ventricular drainage suggests that the initial poor condition was likely the result of acute hydrocephalus or transient elevation of intracranial pressure, while those who did not improve after ventricular drainage more likely had a severe primary brain insult. Designation of clinical grade was made after respiratory and hemodynamic resuscitation, but prior to external ventricular drainage. It is likely that many of those eventually treated actually improved and might therefore no longer have been classified in as poor a grade as they were initially.

Endovascular aneurysm occlusion with coil embolization is less invasive than microsurgical clipping and has been shown to lead to improved outcomes in patients with SAH whose aneurysms are suitable for either technique. ${ }^{5}$ In this series of 33 patients there were two instances in which coiling attempts failed, and two major complications that led to early mortality. Overall, about one-third of the patients treated aggressively did not survive. However, approximately three quarters of the survivors had a good outcome, defined as Modified Rankin Scale score of 2 or less, meaning that they were able to resume functional independence. Only $15 \%$ of those treated with an aggressive approach survived in a poor or dependent condition. Thus, it appears that the selective, aggressive approach taken by the authors has not resulted in a high proportion of devastated survivors.

The Calgary results are consistent with those reported from other contemporary series ${ }^{2-4}$ as well as my own personal experience. After selecting for those who improve after early resuscitation and treatment of acute hydrocephalus, there seems to be a bimodal distribution in terms of cognitive and functional outcomes, with a majority of this small, selected subgroup achieving at least some degree of functional independence. Measures of cognitive functioning and quality of life suggest that these individuals can get along reasonably well in spite of having been hit hard by their bleed. A nihilistic approach to all highgrade SAH patients would deny these patients and their families the potential for recovery.

Many patients with high-grade SAH have suffered irreversible brain injury and will not survive despite aggressive interventions. However, as Diaz and Wong have shown, among these patients there exists a small subgroup with the potential for good recovery. Identification of those with the potential for recovery relies on good clinical judgment and experience. Selection of treatment approach based on the response to initial resuscitation measures seems to be a fairly reliable method to distinguish those for whom aggressive measures would be futile from those with the capacity to recover. In these patients, with such a high proportion recovering to good physical and cognitive function, aggressive treatment efforts are justified.

\author{
Gary Redekop \\ University of British Columbia \\ Vancouver, British Columbia, Canada
}

\section{REFERENCES}

1. Diaz RJ, Wong JH. Clinical outcomes after endovascular coiling in high-grade aneurysmal hemorrhage. Can J Neurol Sci. 2011; 38(1): 30-5.

2. Haug T, Sorteberg A, Finset A, Lindegaard K-F, Lundar T, Sorteberg W. Cognitive functioning and health-related quality of life 1 year after aneurysmal subarachnoid hemorrhage in preoperative comatose patients (Hunt and Hess grade V patients). Neurosurgery. 2010; 66: 475-85.

3. Soehle M, Chatfield D, Czonyka M, Kirkpatrick P. Predictive value of initial clinical status, intracranial pressure, and transcranial Doppler pulsatility after subarachnoid hemorrhage. Acta Neurochir (Wien). 2007; 149: 575-83.

4. van Loon J, Waerzeggars Y, Wilms G, Calenbergh F, Goffin J, Plets C. Early endovascular treatment of ruptured cerebral aneurysms in patients in very poor neurological condition. Neurosurgery. 2002; 50: 457-65.

5. International Subarachnoid Aneurysm Trial (ISAT) Collaborative Group. International Subarachnoid Aneurysm Trial (ISAT) of neurosurgical clipping versus endovascular coiling in 2143 patients with ruptured intracranial aneurysms: a randomised trial. Lancet. 2002; 360: 1267-74. 